\title{
Risk factors for the fever after percutaneous nephrolithotomy: a retrospective analysis
}

\author{
Likun Zhu ${ }^{1,2}$, Rui Jiang ${ }^{1,2}$, Lijun Pei ${ }^{1,2}, \mathrm{Xu} \mathrm{Li}^{1,2}$, Xiangjun Kong ${ }^{1,2}$, Xinwei Wang ${ }^{1,2}$ \\ ${ }^{1}$ Department of Urology Surgery, The Affiliated Hospital of Southwest Medical University, Luzhou 646000, China; ${ }^{2}$ Nephropathy Clinical Medical \\ Research Center of Sichuan Province, Luzhou 646000, China \\ Contributions: (I) Conception and design: L Zhu, R Jiang; (II) Administrative support: L Zhu; (III) Provision of study materials or patients: L Zhu, R \\ Jiang, X Li, X Kong; (IV) Collection and assembly of data: L Zhu, R Jiang, X Li, X Kong; (V) Data analysis and interpretation: L Zhu, X Kong, X \\ Wang; (VI) Manuscript writing: All authors; (VII) Final approval of manuscript: All authors. \\ Correspondence to: Likun Zhu. No. 25, Taiping Street, Jiangyang, Luzhou 646000, China. Email: wuchao198810@163.com.
}

\begin{abstract}
Background: It's very common to see the onset of fever after percutaneous nephrolithotomy (PCNL), it's necessary to analyze the risk factors for the fever following PCNL, and to provide evidence for infection prevention after PCNL.

Methods: A total of 546 adult PCNL patients were included as study subjects and retrospective studies were performed. We collected clinical data of patients using a prospectively designed database. Univariate and multivariate logistic regression analyses were performed to identify the potential risk factors for the fever after PCNL.

Results: Of the included 546 PCNL patients, there were 82 fever patients and 464 no-fever patients following PCNL. Escherichia coli and Proteus mirabilis are the two most common infectious bacteria. Preoperative urinary tract infection $(\mathrm{OR}=4.38,95 \% \mathrm{CI}: 1.15-9.53)$, multiple access $(\mathrm{OR}=5.31,95 \% \mathrm{CI}$ : 1.23-10.75), diabetes $(\mathrm{OR}=4.97,95 \% \mathrm{CI}: 1.37-9.86)$, length of operation $\geq 60 \mathrm{~min}(\mathrm{OR}=5.67,95 \% \mathrm{CI}$ : 2.24-13.42), estimated blood loss in PCNL $\geq 500 \mathrm{~mL}(\mathrm{OR}=2.78,95 \%$ CI: 2.32-3.61) were the independent risk factors associated with postoperative infection.

Conclusions: Effective control of urinary tract infection, reduction of access number, strict control of blood glucose, length of operation control, reduction of intraoperative bleeding should be considered as measures to prevent postoperative fever for patients with PCNL.
\end{abstract}

Keywords: Fever; percutaneous nephrolithotomy (PCNL); risk; retrospective analysis

Submitted Nov 18, 2019. Accepted for publication Mar 16, 2020.

doi: $10.21037 /$ tau.2020.03.37

View this article at: http://dx.doi.org/10.21037/tau.2020.03.37

\section{Introduction}

As a common urological disease, the incidence of urinary tract calculi has significantly increased in recent years, if left untreated, it can severely impair the function of urinary system $(1,2)$. Percutaneous nephrolithotomy (PCNL) has gradually become the preferred choice for the treatment of urinary calculi clinically, and its safety and efficacy have been recognized by patients and clinician (3). However, PCNL can complicate many adverse events. Among them, systemic inflammatory response syndrome (SIRS) is one of the common complications after urinary calculus surgery (4). Even if preoperative prophylactic treatments have been conducted, the incidence of SIRS caused by infection remains rather high. It's been reported $(5,6)$ that 25.8-39.8\% patients get infected after PCNL, and 0.4-3\% sepsis rates were found after PCNL. With consideration to the potentially devastating morbidity and up to $50 \%$ mortality associated with sepsis $(7,8)$, SIRS compromises the effect and safety of PCNL, and it can result in reduced patient comfort, longer hospital stays, and increased medical costs (9). Therefore, the early observations and preventions 
should be established to prevent SIRS.

Fever is one of the most important and timely clinical manifestations of SIRS, the patients with fever may have higher risks of SIRS $(10,11)$. However, the potential reasons and mechanisms related to the onset and development of SIRS remains unclear (12). Furthermore, previous studies $(13,14)$ have indicated that the fever may be a precursor symptom of SIRS, which is an early sign and important factor affecting the prognosis of patients. The observation of fever after PCNL can make important influences on the patients' prognosis. Therefore, it's well-believed that it is necessary to pay attention to the fever after PCNL, and to identify the causes and risk factors of fever to prevent SIRS. Although the factors affecting post-PCNL fever have been extensively evaluated over the years, the main reason for fever and related SIRS after PCNL is not clear. Therefore, in this present study, we retrospectively evaluated the clinical data of patients with fever or no fever following PCNL, aimed to identify the potential risk factors for fever after PCNL, thereby providing evidence for the management of PCNL patients.

\section{Methods}

\section{Ethical considerations}

The study was approved by the Medical Research Ethics Committee of our hospital (No.20170038) and informed consent was taken from all the patients.

\section{Participants}

Between June 2017 and June 2019, a total of 546 adult PCNL patients with completed clinical data were included as study subjects and retrospectively reviewed. The inclusion criteria were as follows: adult patients with age $\geq 18$ years old; they underwent single-stage PCNL and were followed in our hospital; the related clinical data were complete. Patients under the age of 18 , patients with cancer, heart or kidney failure, patients taking immunosuppressive agents, patients taking two or more-stage PCNL, PCNL combined with another surgery in one operation were excluded from this present study.

All the patient data were collected with a prospectively collected database. Patient assessments included medical history, physical examination, urine analysis, midstream urine and blood culture, complete blood count, renal function tests, abdominal plain film, ultrasonography, and/ or drainage urinary tract map. Preoperative abdominal computed tomography was routinely performed in some patients to determine the position of related organs and the proposed nephrostomy tube. All preoperative patients received a single dose of prophylactic antibiotics during the induction of anesthesia.

\section{PCNL procedures}

All the PCNL procedures were performed by the urology surgery team of our hospital. General anesthesia was performed for patients undergoing PCNL. The lithotomy position was taken first, and the ureter was retrogradely inserted into the renal pelvis under the ureteroscope. Then the stent tube was connected with saline to cause artificial hydronephrosis, and the $16 \mathrm{~F}$ double-lumen catheter was placed through the urethra. Then put patients in prone position, the $\mathrm{B}$ ultrasound-guided puncture was performed between the $1^{\text {st }}$ rib or the $12^{\text {th }}$ costal margin or between the posterior tibial line and the lower scapula line, to get into the target renal pelvis, the dilator expanded to $24 \mathrm{~F}$ to introduce the metal sheath and endoscope. The perfusate was $0.9 \% \mathrm{NaCl}$ solution, and the flow rate was controlled by a perfusion pump at $200-350 \mathrm{~mL} / \mathrm{min}$ during the operation, and the ultrasound or pneumatic ballistic lithotripsy was performed. The stone fragments were removed by forceps and irrigation. After the completion of stone removal, one of the F5 double $\mathrm{J}$ tubes in the ureter was placed retrogradely, and $14 \mathrm{~F}$ renal fistula tube was indwelled through the channel. The renal tube was routinely removed 2-4 days after surgery. Antegrade nephrostography was routinely performed before the tube removal to ensure adequate drainage of the collecting system.

\section{Definition of outcomes}

Preoperative urinary tract infection was defined as the bacteria is $\geq 10^{3}$ colony forming units $/ \mathrm{mL}(\mathrm{CFU} / \mathrm{mL})$ in the culture of patients' urinary specimen before PCNL.

The postoperative short-term increase in body temperature below $38.5^{\circ} \mathrm{C}$ was identified as normal response to surgery, and in this present study, the body temperature $\geq 38.5^{\circ} \mathrm{C}$ was defined as fever. For fever patients after PCNL, the blood and urine specimens were both collected and sent for bacteria culture analyses.

\section{Data collection}

We collected the patient's clinical data with prospectively 
designed database: age, gender, underlying disease, preoperative urinary tract infection, hydronephrosis, stone status (position, number, size), operation time, the number of PCNL accesses, intraoperative bleeding, length of catheter removal, length of hospital stay, the results of urinary and blood culture, and postoperative fever.

\section{Statistical analysis}

All of the statistical analyses were analyzed with SPSS 21.0 (SPSS Inc., Chicago, USA). The categorical variables were analyzed using the $\chi^{2}$ test or Fisher's exact test, and the continuous variables were analyzed using Student's t test or Mann-Whitney U test, and it were generally presented as means and standard deviation. Multivariate logistic regression analyses were performed using the forward likelihood ratio selection method to identify independent factors of fever and it is presented with an odds ratio $(95 \%$ confidence intervals, CI). Potential candidate variables were those with $\mathrm{P}<0.05$ in univariate analyses. All of the $\mathrm{P}$ values were 2 tailed, and $\mathrm{P}<0.05$ was considered as being statistically significant.

\section{Results}

\section{The demographic and clinical characteristics of PCNL patients}

The medical records on 546 patients who underwent PCNL were retrospectively collected and analyzed. The overall mean age was $46.3 \pm 9.5$ years old, and there were 418 male patients and 128 female patients. Of the included 546 PCNL patients, there were 82 fever patients and 464 no-fever patients following PCNL. The demographic characteristics and clinical information of PCNL patients are presented in Table 1. There were significant differences in the number of diabetes, preoperative urinary tract infection, PCNL accesses, estimated blood loss in PCNL, length of operation, mean catheter removal between fever and no-fever $\mathrm{PCNL}$ patients (all $\mathrm{P}<0.05$ ), no significant differences were found in the age, gender, hydronephrosis, stone localization, mean stone burden, preoperative creatinine or length of hospital stay between two groups (all $\mathrm{P}>0.05$ ).

\section{Bacterial species detected in the cultures}

The urine culture results of 82 fever patients indicated that there were 21 cases of Escherichia coli, 12 cases of Proteus mirabilis, 1 case of Pseudomonas aeruginosa, 1 case of Klebsiella oxytoca, 3 cases of Enterococcus faecalis, and 44 cases of negative results (Figure 1).

The blood culture results of 82 fever patients indicated that there were 16 cases of Escherichia coli, 7 cases of Proteus mirabilis and 59 cases of negative results (Figure 2).

\section{Multivariate regression analysis on the of fever after PCNL}

An further unconditional logistic multiple regression analysis was further performed on the seven variables with significant differences in univariate analysis, the logistic multiple regression analysis indicated that preoperative urinary tract infection, multiple access, diabetes, length of operation $\geq 60 \mathrm{~min}$, estimated blood loss in PCNL $\geq 500 \mathrm{~mL}$ were independent risk factors associated with postoperative infection respectively (Table 2).

\section{Discussion}

PCNL has gradually become the preferred option for the treatment of most upper urinary calculi in recent years. With the popularity of this technology clinically, more and more complications have been recognized by clinicians. SIRS is one of complications receiving numerous attentions from health care providers, and it's one of the most important and timely clinical manifestations for SIRS $(15,16)$. Therefore, understanding the risk factors affecting postoperative fever after PCNL has important guiding significance for preventing SIRS after PCNL.

The postoperative fever happens frequently in patients with preoperative urinary tract infection, it can be explained by that although antibiotics were used before surgery, the use of the treatment was insufficient and the urinary tract infection was not completely controlled. Besides, for patients with hydronephrosis before surgery, sometimes due to urinary tract obstruction is more serious, after antibiotic treatment, urinary leukopenia. And there may be an illusion that preoperative infection is basically controlled, but the renal pelvis fluid is still turbid and even purulent, and the bacteria in the stones still remain $(3,16)$. Therefore, we believe that patients with preoperative urinary tract infection should be given sufficient attentions, adequate active treatment with sensitive antibiotics to reduce postoperative infection are needed. If the renal sputum is turbid or purulent during surgery, it is necessary to take renal pelvis fluids or stones for bacterial culture and drug 
Table 1 The demographic characteristics and clinical information of PCNL patients

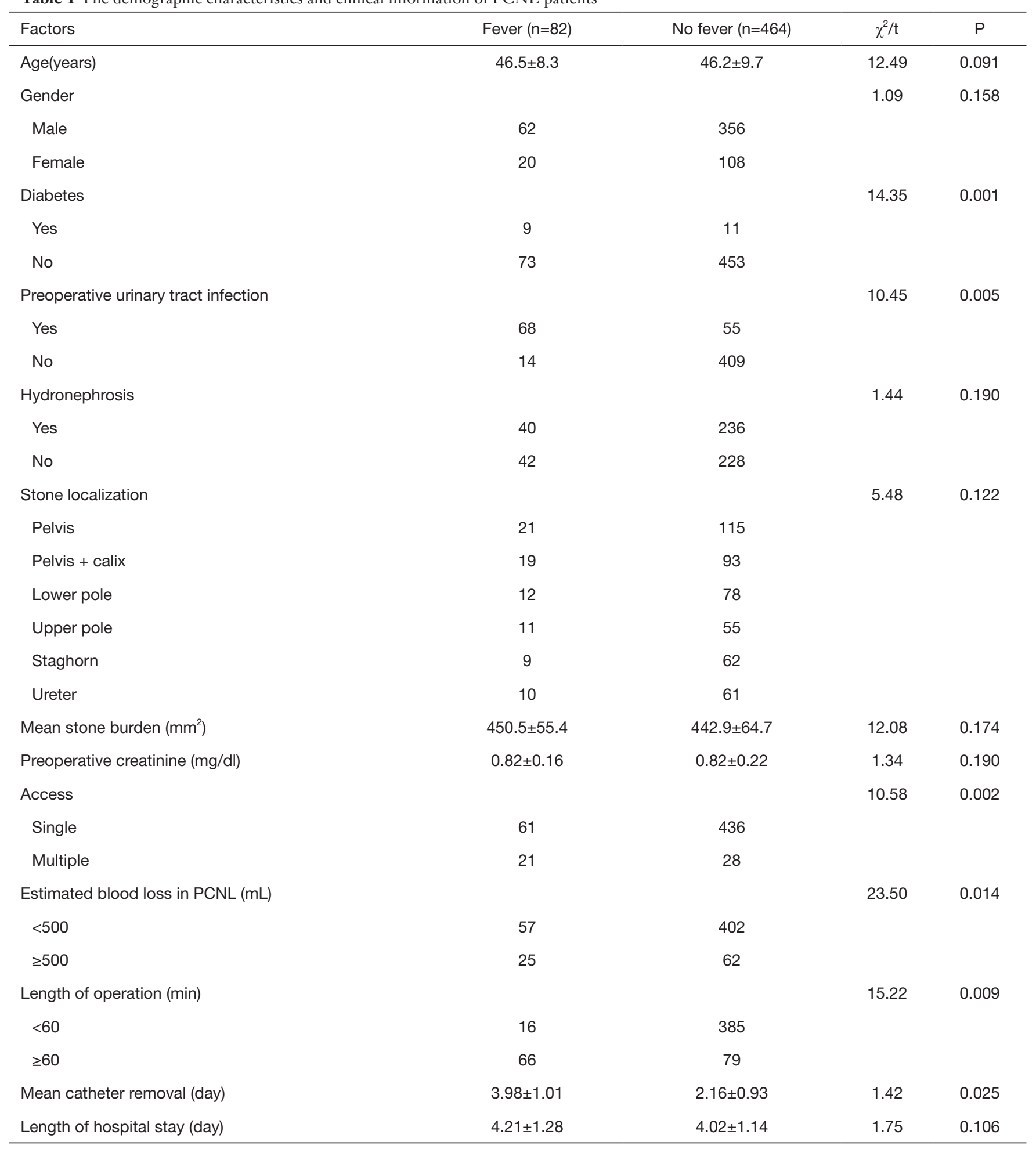




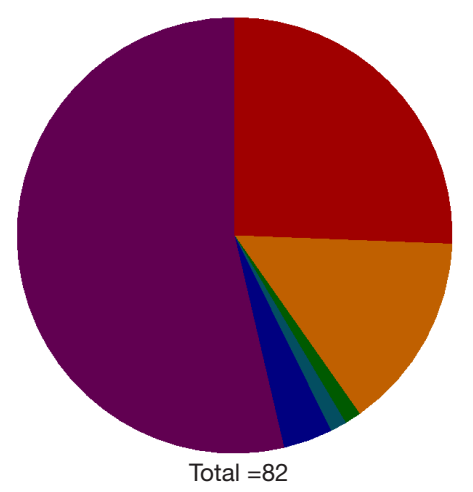

$25.61 \%$ Escherichia coli

$14.63 \%$ Proteus mirab

$1.22 \%$ Pseudomonas aeruginos

$1.22 \%$ Klebsiella oxytoca

$3.66 \%$ Enterococcus faecalis

$53.66 \%$ Negative results

Figure 1 The bacterial distribution of urine culture of 82 fever patients.

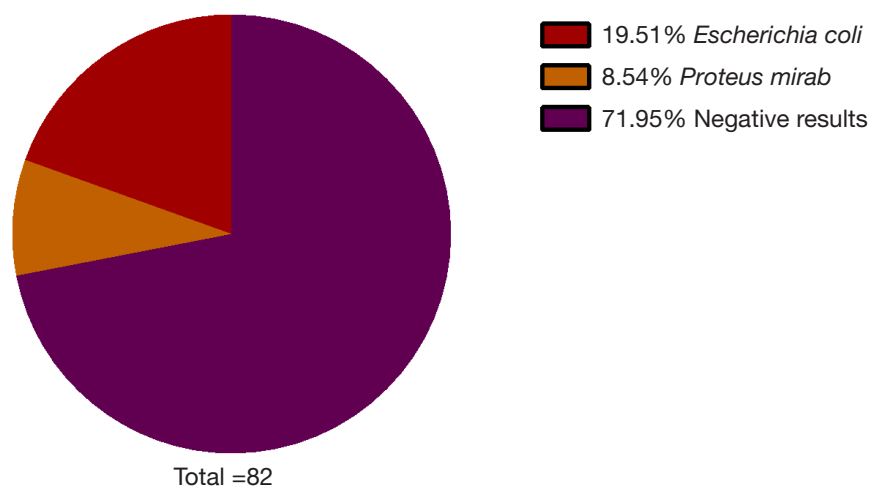

Figure 2 The bacterial distribution of blood culture of 82 fever patients.

Table 2 Multivariate regression analysis on the risk factors of fever after PCNL

\begin{tabular}{|c|c|c|c|c|c|c|}
\hline Factors & $\beta$ & SE & OR & $95 \% \mathrm{Cl}$ & $\mathrm{P}$ & Rank \\
\hline Multiple access & 0.92 & 0.31 & 5.31 & $1.23-10.75$ & 0.031 & 2 \\
\hline Diabetes & 1.03 & 0.45 & 4.97 & $1.37-9.86$ & 0.042 & 3 \\
\hline Length of operation $\geq 60 \mathrm{~min}$ & 0.99 & 0.39 & 5.67 & $2.24-13.42$ & 0.019 & 4 \\
\hline
\end{tabular}

susceptibility test during surgery, so that sensitive antibiotics can be selected for postoperative fever $(17,18)$.

Previous studies (19-21) have reported that the larger the stone, the more gravel, the greater the chance of stone retention, and the higher risk of postoperative infection. Besides, it increases the possibility of damage to the kidney and renal pelvis mucosa during the crushing process, which in turn causes open blood vessels and increased perfusion fluid absorption $(22,23)$. Therefore, many scholars (24-26) at home and abroad believe that the size and quantity of stones are closely related to infection and fever, which are not supported by our findings that the size of stone is not related to the postoperative infection. However, the multiple access is independent risk factors associated with postoperative infection. It's understandable that the larger stones are more difficult to pass through the microchannel gravel, it may be necessary to establish multiple accesses, which accordingly increase the duration of operation time and risk of bleeding $(27,28)$.

Univariate and multivariate logistic regression analyses 
have showed that length of operation $\geq 60 \mathrm{~min}$ and estimated blood loss in PCNL $\geq 500 \mathrm{~mL}$ are risk factors for postoperative fever, with OR values of 5.67 and 2.78, respectively. PCNL requires a large amount of normal fluids to maintain perfusion. Nevertheless, the temperature of normal saline is significantly lower than the body temperature, the fluid can take away many heat from the patient and keep the patient in a low body temperature state (29-31). And the longer the operation, the more serious the situation $(32,33)$. Under the condition of hypothermia, the patient's cardiovascular function changes, coagulation function disorder, which can increase intraoperative blood loss and tissue damage $(34,35)$. Meanwhile, when the operation time is prolonged, the amount of perfusate used can accordingly increase, which will lead to the absorption of a large amount of perfusate (36). Several previous studies (34,37-39) have also found that the amount of fluid absorption in PCNL is positively correlated with the perfusion time. With the damage of the renal pelvis mucosa caused by the crushing process, the perfusate easily flows into the blood through the renal sputum, and bacteria or toxins can enter the blood by this pathway easily. The longer the time, the more substances that enter the bloodstream, the greater the possibility of postoperative infection (40). Especially for patients with severe blood vessel damage and massive intraoperative bleeding, the operation should be stopped decisively, the renal fistula should be placed and the hemostasis should be clamped, and the calculus should be treated after 1-2 weeks.

Several limitations must be considered in this present study. Firstly, previous study (41) has reported that the postoperative infection is strongly associated with A. baumannii bacteria. Limited by sample size, this present study did not analyze bacterial species and infection correlation. Secondly, we didn't construct a model for predicting the infection after PCNL, which can provide more reference value for clinical use. With more sample included in the future, it's necessary to develop a prediction model for infection prevention after PCNL.

In conclusion, patients with preoperative urinary tract infection, multiple access, diabetes, length of operation $\geq 60 \mathrm{~min}$, estimated blood loss in PCNL $\geq 500 \mathrm{~mL}$ potentially risk more for postoperative infection after PCNL. Still, there may be many other factors involved in fever following PCNL that warrants further investigations. Effective control of urinary tract infection, reduction of access number, strict control of blood glucose, length of operation control, reduction of intraoperative bleeding must be highlighted for the management of patients with PCNL.

\section{Acknowledgments}

Funding: This study received grants from Sichuan Province Health Department (16158).

\section{Footnote}

Conflicts of Interest: All authors have completed the ICMJE uniform disclosure form (available at http://dx.doi. org/10.21037/tau.2020.03.37). The authors have no conflicts of interest to declare.

Ethical Statement: The authors are accountable for all aspects of the work in ensuring that questions related to the accuracy or integrity of any part of the work are appropriately investigated and resolved. The study was approved by the Medical Research Ethics Committee of our hospital (No.20170038) and informed consent was taken from all the patients.

Open Access Statement: This is an Open Access article distributed in accordance with the Creative Commons Attribution-NonCommercial-NoDerivs 4.0 International License (CC BY-NC-ND 4.0), which permits the noncommercial replication and distribution of the article with the strict proviso that no changes or edits are made and the original work is properly cited (including links to both the formal publication through the relevant DOI and the license). See: https://creativecommons.org/licenses/by-nc-nd/4.0/.

\section{References}

1. Yamashita S, Kohjimoto Y, Hirabayashi Y, et al. Upper urinary tract stone disease in patients with poor performance status: active stone removal or conservative management? BMC Urol 2017;17:103.

2. Tian Y, Yang X, Luo G, et al. Initial prospective study of ambulatory $\mathrm{mPCNL}$ on upper urinary tract calculi. Urol J 2020;17:14-8.

3. Li D, Sha ML, Chen L, et al. Is the Preoperative Level of Procalcitonin a Valid Indicator for Predicting Postoperative Fever After Percutaneous Nephrolithotomy? J Endourol 2018;32:192-7.

4. Proietti S, Giusti G, Desai M, et al. A Critical Review of Miniaturised Percutaneous Nephrolithotomy: Is Smaller Better? Eur Urol Focus 2017;3:56-61. 
5. Cetinkaya M, Buldu I, Kurt O, et al. Platelet-toLymphocyte Ratio: A New Factor for Predicting Systemic Inflammatory Response Syndrome after Percutaneous Nephrolithotomy. Urol J 2017;14:4089-93.

6. Omar M, Noble M, Sivalingam S, et al. Systemic Inflammatory Response Syndrome after Percutaneous Nephrolithotomy: A Randomized Single-Blind Clinical Trial Evaluating the Impact of Irrigation Pressure. J Urol 2016;196:109-14.

7. Taniguchi LU, Pires EMC, Vieira JM, Jr., et al. Systemic inflammatory response syndrome criteria and the prediction of hospital mortality in critically ill patients: a retrospective cohort study. Rev Bras Ter Intensiva 2017;29:317-24.

8. Churpek MM, Zadravecz FJ, Winslow C, et al. Incidence and Prognostic Value of the Systemic Inflammatory Response Syndrome and Organ Dysfunctions in Ward Patients. Am J Respir Crit Care Med 2015;192:958-64.

9. Rudd KE, Seymour CW, Aluisio AR, et al. Association of the Quick Sequential (Sepsis-Related) Organ Failure Assessment (qSOFA) Score With Excess Hospital Mortality in Adults With Suspected Infection in Low- and Middle-Income Countries. JAMA 2018;319:2202-11.

10. Liao MM, Lezotte D, Lowenstein SR, et al. Sensitivity of systemic inflammatory response syndrome for critical illness among ED patients. Am J Emerg Med 2014;32:1319-25.

11. Kaygisiz O, Satar N, Gunes A, et al. Factors predicting postoperative febrile urinary tract infection following percutaneous nephrolithotomy in prepubertal children. J Pediatr Urol 2018;14:448 e1- e7.

12. Alazawi W, Pirmadjid N, Lahiri R, et al. Inflammatory and Immune Responses to Surgery and Their Clinical Impact. Ann Surg 2016;264:73-80.

13. Garami A, Steiner AA, Romanovsky AA. Fever and hypothermia in systemic inflammation. Handb Clin Neurol 2018;157:565-97.

14. Li T, Sun XZ, Lai DH, et al. Fever and systemic inflammatory response syndrome after retrograde intrarenal surgery: Risk factors and predictive model. Kaohsiung J Med Sci 2018;34:400-8.

15. Swonke ML, Mahmoud AM, Farran EJ, et al. Early Stone Manipulation in Urinary Tract Infection Associated with Obstructing Nephrolithiasis. Case Rep Urol 2018;2018:2303492.

16. Lai WS, Assimos D. Factors Associated With Postoperative Infection After Percutaneous Nephrolithotomy. Rev Urol 2018;20:7-11.
17. Chew BH, Miller NL, Abbott JE, et al. A Randomized Controlled Trial of Preoperative Prophylactic Antibiotics Prior to Percutaneous Nephrolithotomy in a Low Infectious Risk Population: A Report from the EDGE Consortium. J Urol 2018;200:801-8.

18. Parkhomenko E, De Fazio A, Tran T, et al. A MultiInstitutional Study of Struvite Stones: Patterns of Infection and Colonization. J Endourol 2017;31:533-7.

19. Koras O, Bozkurt IH, Yonguc T, et al. Risk factors for postoperative infectious complications following percutaneous nephrolithotomy: a prospective clinical study. Urolithiasis 2015;43:55-60.

20. Hu M, Zhong X, Cui X, et al. Development and validation of a risk-prediction nomogram for patients with ureteral calculi associated with urosepsis: A retrospective analysis. PLoS One 2018;13:e0201515.

21. Nouralizadeh A, Pakmanesh H, Basiri A, et al. Percutaneous nephrolithotomy of staghorn renal stones in pediatric patients using adult-sized instrument. Urologia 2019;86:211-5.

22. Rivera M, Viers B, Cockerill P, et al. Pre- and Postoperative Predictors of Infection-Related Complications in Patients Undergoing Percutaneous Nephrolithotomy. J Endourol 2016;30:982-6.

23. Bai Y, Tang Y, Deng L, et al. Management of large renal stones: laparoscopic pyelolithotomy versus percutaneous nephrolithotomy. BMC Urol 2017;17:75.

24. Kreydin EI, Eisner BH. Risk factors for sepsis after percutaneous renal stone surgery. Nat Rev Urol 2013;10:598-605.

25. Eswara JR, Lee H, Dretler SP, et al. The effect of delayed percutaneous nephrolithotomy on the risk of bacteremia and sepsis in patients with neuromuscular disorders. World J Urol 2013;31:1611-5.

26. Kim BS. Recent advancement or less invasive treatment of percutaneous nephrolithotomy. Korean J Urol 2015;56:614-23.

27. Perez-Fentes D. [Techniques for percutaneous access during percutaneous nephrolithotomy.]. Arch Esp Urol 2017;70:155-72.

28. Rassweiler J, Rassweiler MC, Klein J. New technology in ureteroscopy and percutaneous nephrolithotomy. Curr Opin Urol 2016;26:95-106.

29. Zhong $\mathrm{W}$, Zeng $\mathrm{G}, \mathrm{Wu} \mathrm{K}$, et al. Does a smaller tract in percutaneous nephrolithotomy contribute to high renal pelvic pressure and postoperative fever? J Endourol 2008;22:2147-51

30. Hosseini SR, Mohseni MG, Aghamir SMK, et al. Effect 
of Irrigation Solution Temperature on Complication of Percutaneous Nephrolithotomy: A Randomized Clinical Trial. Urol J 2019;16:525-9.

31. Kati B, Buyukfirat E, Pelit ES, et al. Percutaneous Nephrolithotomy with Different Temperature Irrigation and Effects on Surgical Complications and Anesthesiology Applications. J Endourol 2018;32:1050-3.

32. Mirza S, Panesar S, AuYong KJ, et al. The effects of irrigation fluid on core temperature in endoscopic urological surgery. J Perioper Pract 2007;17:494-7, 9-503.

33. Yu J, Guo B, Yu J, et al. Antibiotic prophylaxis in perioperative period of percutaneous nephrolithotomy: a systematic review and meta-analysis of comparative studies. World J Urol 2019. [Epub ahead of print].

34. Saxena D, Sapra D, Dixit A, et al. Effects of fluid absorption following percutaneous nephrolithotomy: Changes in blood cell indices and electrolytes. Urol Ann 2019;11:163-7.

35. Singh R, Asthana V, Sharma JP, et al. Effect of irrigation fluid temperature on core temperature and hemodynamic changes in transurethral resection of prostate under spinal anesthesia. Anesth Essays Res 2014;8:209-15.

36. Li D, Sha M, Chen L, et al. A preliminary study: the role of preoperative procalcitonin in predicting postoperative

Cite this article as: Zhu L, Jiang R, Pei L, Li X, Kong X, Wang $X$. Risk factors for the fever after percutaneous nephrolithotomy: a retrospective analysis. Transl Androl Urol 2020;9(3):1262-1269. doi:10.21037/tau.2020.03.37 fever after mini-percutaneous nephrolithotomy in patients with a negative baseline urine culture. Urolithiasis 2019;47:455-60.

37. Guzelburc V, Balasar M, Colakogullari M, et al. Comparison of absorbed irrigation fluid volumes during retrograde intrarenal surgery and percutaneous nephrolithotomy for the treatment of kidney stones larger than $2 \mathrm{~cm}$. Springerplus 2016;5:1707.

38. Mohta M, Bhagchandani T, Tyagi A, et al. Haemodynamic, electrolyte and metabolic changes during percutaneous nephrolithotomy. Int Urol Nephrol 2008;40:477-82.

39. Zhang B, Xie H, Hu Y, et al. The visual percutaneous nephrolithotomy versus the conventional percutaneous nephrolithotomy in treatment for renal stone. Minerva Urol Nefrol 2019;71:627-35.

40. Amri M, Naouar S, Ben Khalifa B, et al. Predictive factors of bleeding and fever after percutaneous nephrolithotomy. Tunis Med 2019;97:667-74.

41. Al-Gethamy MM, Faidah HS, Adetunji HA, et al. Risk factors associated with multi-drug-resistant Acinetobacter baumannii nosocomial infections at a tertiary care hospital in Makkah, Saudi Arabia - a matched case-control study. J Int Med Res 2017;45:1181-9. 